\title{
Research on Efficacy of Using Social Networks in Academic Teaching Metrics, Experiments, and Results
}

\author{
Maria Grazia Albanesi ${ }^{1}$, Riccardo Amadeo ${ }^{1}$, Giovanni Vecchio ${ }^{1}$
}

\begin{abstract}
The study integrates a customized private social network in an academic course. We propose and measure a new metric, based on gamification principles, to understand the benefits on the student learning process. We applied the idea to two academic classes of 25 and 80 students, respectively, (Master Degree in Economy and Engineering Faculties, University of Pavia). The social networks has been monitored for one semester. The paper describes the educational principle at the basis of the methodology, the developed metric, and the results. This case study shows that the introduction of innovative approaches to social networking in academic classes can be used to focus student attention, rise motivation and, as final goal, increase the level of knowledge.
\end{abstract}

Keywords: Academic Education, Social Networking, gamification.

\section{Introduzione}

La nascita delle tecnologie alla base del Web 2.0 è stata una delle principali innovazioni dell'informatica, in quanto ha avuto parecchie applicazioni e ricadute in campo sociale(Lin \& Lu, 2011), economico (DiMicco, et al., 2008)e politico(Tsui, Lee, \& Yao, 2010). In particolare, i social network hanno introdotto nuovi paradigmi di comunicazione tra gli utenti, divenendo un fenomeno studiato da psicologi ed esperti della comunicazione ( $\mathrm{Lin} \& \mathrm{Lu}, 2011)$. Non stupisce quindi che esistano molti tentativi di mettere a disposizione i social network anche nel campo della didattica (Erenli, 2012), pur con differenti obbiettivi. Per una corretta categorizzazione dei vari contributi in letteratura, occorre fare distinzione tra i termini disocial media e social network. Entrambi basati sulla medesima tecnologia abilitante (Internet e il Web), nei social media si preferisce privilegiare la diffusione dell'informazione, di tipo ipermediale, con una media o bassa interattività, mentre nei social network si privilegia l'aspetto di interconnessione delle relazioni sociali tra $\mathrm{i}$ membri del social network, con una forte connotazione di interattività e partecipazione. Questa distinzione, seppure ben presente in letteratura (Boyd \& Ellison, 2007), (Kaplan \& Haenlein, 2010),(Lin, Li, Wang, Salamantian, \& Xie, 2012) viene spesso trascurata e i due termini finiscono per essere usati indifferentemente. Tuttavia, è chiaro che non si tratta solo di una scelta di terminologia, in quanto i due concetti sono abbastanza differenti.

Nel progetto documentato nel presente articolo, la scelta è stata quella di realizzare un social network ad hoc, pensato per una classe accademica di studenti al secondo livello di 
laurea (Laurea Magistrale). La prima obiezione possibile a questa scelta è per quale ragione non si è utilizzato uno dei tanti social network liberamente disponibili in rete, come per esempio la scelta più ovvia e diffusa nel nostro paese di Facebook, invece di utilizzare l'implementazione personalizzata. Sono molteplici le ragioni che hanno portato ad escludere l'uso di un social network esterno/pubblico all'organizzazione accademica. Ecco di seguito le principali:

1. Le politiche di privacy di un social network esterno generalmente sono al di fuori del controllo dell'amministratore (il docente). Possono essere configurate, ma i principi base difficilmente possono essere modificati. Inoltre, ciò che più grave, sono soggette a modifiche unilaterali (si pensi alle polemiche spesso generate dai cambiamenti di policy di privacy di Facebook (Debatin, Lovejoy, Horn, \& Hughes, 2009).

2. Nella struttura di classe virtuale, si vuole comunque conservare la gerarchia docentestudenti presente nella classe reale, senza però limitare la libertà dei singoli alla creazione di aree riservate, sottogruppi, in piena autonomia. Ciò non sempre è possibile con il solo supporto della distinzione tra amministratore e utente. Due soli livelli di gerarchia, tipici dei principali social network, non sono sufficienti a garantire, nello stesso tempo, il controllo del docente e una certa autonomia organizzativa degli studenti, fondamentale in una didattica a livello universitario. Questa scelta è ulteriormente rafforzata dal fatto che la classe virtuale creata coincide con quella reale, in quanto non vuole essere un'alternativa, ma un'aggiunta, uno strumento in più per catalizzare la classe reale attorno a obiettivi comuni.

3. Risulta utile prevedere meccanismi di protezione delle informazioni pubblicate sul social network, al fine di rispettare le leggi sui copyright e di accesso limitato alle informazioni. E' pertanto necessario prevedere la funzione di login con password in particolari sotto - parti del social network, cosa non sempre disponibile su social network esterni.

4. La possibilità di fare backup totali o incrementali di tutto il materiale pubblicato sul social network permette una migliore gestione delle informazioni e assicura il completo controllo della stessa. Questa funzione non sempre è a disposizione degli utenti di social network esterni.

5. La maggior parte di social network esterni, pur basandosi su database di dati (di tipo SQL o non SQL), non permettono la creazione di database accessibili da parte dell'amministratore del social network. Ciò può essere una limitazione, perché la possibilità di strutturare l'informazione può rivelarsi utile al crescere del numero di studenti o per separare i dati da un anno accademico all'altro.

Per tutti questi motivi, si è deciso di creare un social network ad hoc ospitato su un server del Dipartimento di Ingegneria Industriale e dell'Informazione, a cui lo staff del progetto ha completo accesso con i massimi privilegi di amministrazione, sia sul social network stesso sia sul sistema operativo del server. I dettagli tecnici della soluzione realizzata sono descritti nel paragrafo 3. Chiameremo il social network realizzato ad hoc Course Social Network (CSN), dal nome del progetto stesso (Course Social Network Project 2013-2014).

\section{Principi educativi alla base del progetto}

L'introduzione della tecnologia di social networking nella metodologia di insegnamento a livello accademico ha ovviamente lo scopo ultimo di migliorare i risultati 
di apprendimento degli studenti. Tuttavia, questo obiettivo può essere raggiunto seguendo diversi principi educativi, molti dei quali anche controversi, che provengono da differenti discipline. E' necessario quindi, per distinguere un esperimento di didattica da un altro, non solo analizzare la sua implementazione e i risultati, ma anche decidere a priori quali sono i principi educativi alla base dell'esperimento stesso, e come si vogliono implementare utilizzando la tecnologia scelta (in questo caso quella dei social network). Un primo principio educativo ampiamente condiviso presuppone di prendere in considerazione le conoscenze pregresse degli studenti. Dal momento che i corsi oggetto della sperimentazione sono due classi di Laurea Magistrale, la popolazione degli studenti è particolarmente eterogenea, poiché i curricula pregressi non sono affatto simili: molti provengono da corsi triennali diversi, anche da diverse Università, altri hanno alle spalle un'attività lavorativa, i più anche all'estero. Pertanto non è stato possibile fare alcuna ipotesi sulle conoscenze pregresse degli studenti; pur sapendo che questo fattore può influenzare notevolmente l'apprendimento, non è stato preso in considerazione poiché non è previsto un test d'ingresso o una valutazione preliminare delle conoscenze degli studenti, se non per il solo voto di laurea.

L'organizzazione del materiale di studio è fondamentale per migliorare l'apprendimento: a questo scopo sono stati utilizzate sul Course Social Network delle pagine di approfondimento per creare link tra argomenti trattati a lezione, seminari proposti e progetti realizzati dagli studenti. Ovviamente, in questa organizzazione dell'informazione, sono stati ampiamente sfruttati gli aspetti tecnologici propri del Web 2.0 di collegamenti ipermediali, repository di dati digitali (articoli, presentazioni, video) e post di Blog.

Un aspetto importante dell'utilizzo dei social network nella didattica riguarda il principio di sostenere la motivazione degli studenti (Ford, 1992)Non sempre la motivazione degli studenti è abbastanza alta da massimizzare il processo di apprendimento. Le cause di mancanza di motivazione possono essere essenzialmente due: la prima è legata alla personalità dello studente, dotato di scarsa volontà anevrotica,che non riesce a sostenere lo sforzo di apprendimento quando non intravede un possibile vantaggio o interesse per la materia, mentre la seconda è più legata all'efficacia del metodo di insegnamento (maggiore coinvolgimento, presentazione degli argomenti nell'ordine corretto, chiarezza e originalità dell'esposizione). Nel primo caso, possono venire in aiuto le tecniche di gamification, che alzano la motivazione dello studente che si vede premiato per la sua partecipazione al processo di apprendimento. Nel secondo caso, l'organizzazione del materiale di studio e il coinvolgimento in attività pratiche sono molto utili. Per quanto riguarda questo secondo aspetto, infatti, è possibile innalzare la partecipazione pratica mediante la proposta sul Course Social Network di esercizi, progetti, ricerca di informazioni, presentazioni da effettuare in aula. Con l'utilizzo del Blog, il docente può incoraggiare le attività pratiche collaterali proponendo le attività in modo anche completamente asincrono rispetto alle lezioni frontali in aula.

Un altro principio educativo importante è quello di ottenere feedback da parte degli studenti: analizzare $\mathrm{i}$ post degli studenti alla ricerca di giudizi di apprezzamento, o deprezzamento, perplessità, dubbi, permette di ritornare su argomenti che sono stati trattati e migliorare l'efficacia della trasmissione del messaggio didattico. Senza un social network, che memorizza messaggi e commenti degli studenti, questo feedback sarebbe molto meno efficace, limitato a interazioni informali al termine delle lezioni.

Infine l'ultimo principio educativo alla base del nostro approccio è considerarlo stato emotivo e sociale, oltre che intellettuale, degli studenti. L'enorme esperienza che hanno 
gli studenti di oggi, nativi digitali, di interagire in rete, permette di sfruttare questa confidenza nel mezzo di comunicazione e l'abitudine ad esprimere anche aspetti emotivi e sociali della propria personalità. Occorre infatti ricordare che uno studente non è solo un'entità intellettuale, ma ha anche una dimensione sociale ed emotiva che influisce enormemente sul processo di apprendimento. Molti studi hanno dimostrato che il clima creato all'interno della classe ha una evidente ricaduta sull'apprendimento. Un clima negativo può limitare le prestazioni, mentre un clima positivo può entrare in sinergia e amplificare l'apprendimento. Con l'utilizzo del social network è possibile rafforzare la tendenza ad esprimere emozioni e relazioni sociali positive (per esempio con il meccanismo ben noto dei Like), per costruire un clima del Course Social Network in grado di influenzare positivamente anche la classe reale.

\section{Obiettivi e motivazioni della soluzione proposta}

Per trarre i massimi vantaggi dal modello di social network, al fine permette di sfruttare tutte le sue potenzialità, gli obiettivi principali nel nostro approccio sono stati i seguenti:

1. Creare un ambiente di classe virtuale in cui gli appartenenti si sentano saldamente parte della comunità, al pari di una classe reale.

2. Stimolare la partecipazione e la condivisione di idee, materiale creato dagli studenti, secondo il paradigma di User Generated Content (UGC)(Leung, 2009).

3. Utilizzare il paradigma di crowdsourcing per ottenere idee, materiale o progetti realizzati per un eventuale aggiornamento continuo del programma del corso.

4. Stimolare e incoraggiare la creazione spontanea di gruppi del social network. Questa facility è praticamente presente in quasi tutte le piattaforme tecnologiche di creazione di social network, ma la maggior parte delle volte, anche laddove presente, i gruppi sono comunque gestiti dall'amministratore e non dai singoli. La formazione di un sottogruppo lasciata come libera iniziativa ai singoli membri è invece prerogativa di piattaforme personalizzabili di social network, e questa possibilità consente agli studenti di organizzarsi attorno ai loro interessi per un determinato argomento del corso o attività progettuale.

5. Premiare la partecipazione con una valutazione oggettiva, mediante metriche e tecniche di gamification, come verrà spiegato nei paragrafi successivi.

Essendo i punti 1-5 i principali criteri di utilizzo del social network adottati nel presente studio, la scelta della piattaforma tecnologica è stata WordPress, uno dei più conosciuti Content Managment System gratuiti (licenza GNU general public license). Questo CMS è sviluppato in PHP e che si basa su database MySQL. Si è scelto WordPress principalmente per la sua semplicità di gestione: implementare le funzionalità desiderate è stato molto semplice, grazie alla struttura modulare del sistema. Inoltre, per la larghissima diffusione dell'utilizzo di WordPress nel web, abbiamo ipotizzato che gli utenti del sistema avrebbero avuto minori difficoltà disabilità, in quanto il nostro portale avrebbe ricalcato lo stile di utilizzo già proprio della loro esperienza di navigazione Web.Abbiamo creato il nostro portale a partire dalla versione di WordPress3.6.1, integrata da plug-in scelti ad-hoc per ricreare lo stile di un social network di uso comune con meccaniche di gamification. Il principale plug-in utilizzato è BuddyPress 1.8.1, strumento primario utilizzato per offrire agli iscritti al sistema le potenzialità di un social network (profilo con possibilità di inserire dati personali e foto, bacheca, possibilità di scambiare messaggi privati e di creare 
o iscriversi a gruppi, possibilità di avere una cosiddetta "activity stream", cioè una storia delle attività compiute sul sistema). Il secondo componente aggiuntivo rilevante è invece il plug-in deputato all'implementazione della gamification. La scelta è ricaduta su CubePoints 3.2.1, insieme alla sua integrazione per BuddyPress (CubePointsBuddypressIntegration). Sono stati inoltre i seguenti plug-in, per avere una più semplice gestione del sistema e per raccogliere le statistiche di utilizzo dello stesso:Are You A Human, New User Approve, TR RegisterOnly(tutti e tre utili per gestire la registrazione e l'accesso ai contenuti da parte di utenti, iscritti e non), AdminCommentersCommentCount, Counterize, JetPack, per la raccolta di statistiche sul traffico web. In aggiunta a questi, in seconda battuta è stato aggiunto anche GD Star Rating, un plug-in che permette di far valutare dagli utenti ogni contenuto creato sul sistema con una scala numerica scelta dall'amministratore dello stesso. L'utilità di questa aggiunta verrà chiarita nel paragrafo 5 .

\section{La principale innovazione: la gamification per la didattica}

Il concetto di gamification si è sviluppato in tempi piuttosto recenti. Proprio per questo, la sua definizione non è ancora stata formulata in modo formale e condiviso da tutta la comunità scientifica. Gli studi(Hamari J. , 2013), (Tuunanen \& Hamari, 2014)definiscono la gamification come l'introduzione in servizi esistenti di strumenti intuitivi che ricreino un'esperienza ludica nell'utente. In maniera ancora più concisa,Deterding et al.(Deterding, Dixon, Khaled, \& Nacke, 2011) riducono la gamification alla semplice "inclusione di elementi ludici in contesti non ludici". Huotari and Hamari(Huotari \& Hamari, 2012), allargano la definizione includendo nella stessa lo scopo della gamification, che diventa un mezzo per "supportare la creazione di valore complessivo per l'utente". Comunque si voglia definire, il concetto di gamification rimane piuttosto intuitivo: una tecnica motivazionale che nasce con lo scopo di rendere più stimolante lo svolgimento di alcuni compiti o l'acquisizione di alcune informazioni. L'idea di base è quella di aggiungere aspetti di sfida o di divertimento a sistemi informatici pensati per l'utilizzo degli utenti, al fine di migliorare la loro esperienza interattiva con tale sistema.

L'utilizzo di tecniche di gamification in ambiti di apprendimento accademico ha iniziato a diffondersi recentemente, con risultati altalenanti. Lo studio in proposto da K. Berkling e C. Thomas (Berkling \& Thomas, 2013), ad esempio, propone un utilizzo della gamification in un insegnamento universitario di Software Engineering. La componente ludica, in questo caso, è stata implementata in tutti gli aspetti dell'insegnamento, costringendo quindi gli studenti a partecipare alle attività tipiche di un approccio di gamification. A seguito di ciò la componente di "volontarietà" della partecipazione è andata persa(Mcgonigal, 2011). I risultati di tale studio, che includono questionari soggettivi tra i partecipanti, sono purtroppo stati negativi relativamente agli obbiettivi posti (aumentare il coinvolgimento degli studenti e la loro motivazione per mezzo di attività di apprendimento indipendenti e flessibili). Le valutazioni soggettive raccolte a posteriori tra gli studenti dimostrano come la trasformazione del corso tramite gamification non sia stata apprezzata dagli studenti. All'opposto, G. Barata et alter (Barata, Gama, Jorge, \& Goncalves, 2013)propongono uno studio che ha portato ad ottimi risultati: il contesto, anche in questo caso, è quello universitario, e il sistema costruito è stato proposto ad una classe tecnica. I feedback degli studenti sono stati molto più positivi, rispetto al primo studio presentato. Le opinioni raccolte definiscono un quadro nel quale risalta l'elevata 
motivazione allo studio (rispetto ad altri corsi) indotta dall'introduzione dell'elemento ludico. Metriche oggettive, inoltre, (presenze alle lezioni, partecipazione online, utilizzo del materiale di studio) quantificano un aumento dell'impegno degli studenti rispetto al medesimo insegnamento proposto senza gamification.

Nonostante l'ampia gamma di possibilità di utilizzo e il recente avvento della disciplina, l'implementazione di meccanismi di gamification in applicazioni informatiche, specialmente web, ha una struttura piuttosto consolidata. Gli elementi cardine, conosciuti anche come motivationalaffordances(Hamari, Koivisto, \& Harri, 2014)sono dieci:

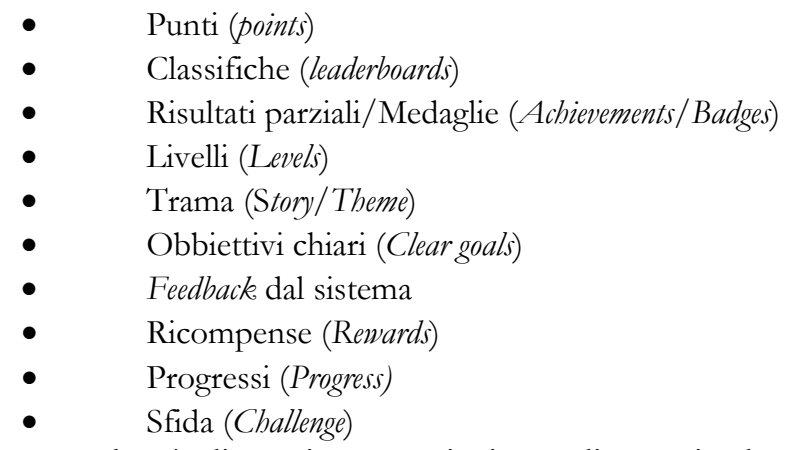

La volontà di motivare un insieme di utenti ad effettuare un'azione (nel nostro caso, apprendere; in casi più generali, acquistare, condividere le proprie conoscenze, i propri interessi e molti altri) avviene tramite sfide con obbiettivi chiari, che se superate ricompensano l'utente tramite punti che permettono una progressione o nella storia (se esiste una componente narrativa) o in una (o più) classifica/e. A tutto ciò il sistema somma continui feedback all'utente relativi ai suoi progressi sia nel gioco che nell'attività di apprendimento ("Hai ottenuto $n$ punti e hai imparato la nozione prevista").

\section{Realizzazione del Course Social Network}

Una delle caratteristiche di un Social Networkè l'uniformità dei privilegi, delle opzioni e delle possibilità date a ciascun utente. Nel nostro sistema, invece, la presenza del docente e le sue attività sono state volutamente evidenziate. Al docente è stata data la possibilità di creare contenuti (post) che dettassero i temi principali proposti sul social network di settimana in settimana, traendo spunto dalle lezioni frontali. Agli studenti, è stata data la possibilità di iscriversi al Social Network (partecipazione volontaria) per commentare e sviluppare una discussione su temi scelti dal docente o per creare contenuti personali. Questa descrizione ci porta a definire il nostro sistema come un ibrido tra un blog e un Social Network classico, architettura che, a nostro avviso, si presta meglio per integrare un insegnamento universitario.

Course Social Network fa ampio uso della gamification. Nel progetto della piattaforma è stata seguita la struttura base descritta nel paragrafo precedente. Abbiamo quindi creato un sistema che prevede ricompense per gli studenti che compiono azioni "costruttive" (vale a dire indirizzate all'apprendimento). Tali ricompense sono punti interni al sistema, definiti punti CSN. Dopo aver sostenuto l'esame alla fine dell'insegnamento ed aver ottenuto la relativa valutazione, l'accumulo di punti su CSN si trasforma in un aumento proporzionale della stessa, trasformando gli astratti punti CSNin concreti bonus sul voto finale del test. 
Come definire cosa sia un'azione costruttiva al fine di rendere efficace l'insegnamento ha portato alla creazione di una corrispondenza tra azione effettuata e premio in punti CSN. Le azioni premiate sono state definite come segue: l'iscrizione al sistema, il log-in giornaliero, la creazione di un gruppo di discussione, la partecipazione ad un gruppo creato da altri, la partecipazione ad una discussione proposta dal docente tramite commento, la creazione di un link con un altro utente, lo scambio di messaggi privati. A queste attività si aggiunge la possibilità per il docente di premiare arbitrariamente gli studenti per lo svolgimento meritevole di attività proposte.

Per implementare l'aspetto competitivo tipico di una struttura ludica è stata introdotta una classifica basata sui punti ricevuti. Tale classifica è stata resa sempre visibile ad ogni utente, insieme al suo "livello" di partecipazione all'attività. L'accumulo di punti porta ad aumentare il proprio livello, passando da "iscritto" a "lettore" a "partecipante", a seconda della quantità di punti accumulata. Le soglie di passaggio sono state decise prima dell'inizio del progetto. Il bonus alla valutazione d'esame è stato pensato, a scalare, per i primi della classifica.

Il principale problema che questo approccio può portare è la difficoltà, soprattutto al crescere dei contenuti creati dagli utenti, nel distinguere i contenuti "di qualità" (vale a dire creati col fine primario di contribuire ad una discussione) da quelli creati col mero scopo di accumulare punti e aumentare il proprio livello (spamming). Per ovviare a ciò è stato prima di tutto spiegato agli studenti che si presuppone la loro "buona fede" nell'utilizzo del sistema. In seconda battuta, è stata implementata una policy di peerrevienving dei contenuti creati dagli utenti, per mezzo del plug-in GD Star Rating menzionato in precedenza. Ogni contenuto creato da uno studente (eccettuati i messaggi privati, illeggibili anche all'amministratore di sistema) può essere valutato tramite una scala a cinque punti da ogni altro utente iscritto al sistema. I commenti a bassa valutazione sono messi in risalto al gestore (il docente) che può decidere se e quali provvedimenti prendere. Ovviamente, i provvedimenti sono pensati solo per evidenti tentativi di accumulare punti tramite pratiche scorrette e non per valutare i concetti espressi dagli utenti nei contenuti. Questo sistema ha portato ad un effetto imprevisto: pensato per garantire la correttezza della "gara" non ha segnalato alcun abuso delle meccaniche ludiche, ma in più occasioni ha anzi portato all'attenzione del docente contributi molto validi, facendo in modo che il docente stesso premiasse ulteriormente il merito del commento. La meccanica dimostra l'utilizzo in buona fede del sistema da parte degli studenti: pur sapendo che la segnalazione di contributo di qualità avrebbe premiato un "concorrente" in termini di classifica, gli utenti hanno comunque scelto di dare merito e risalto a un collega agendo contro il loro interesse numerico ma a favore dell'interesse accademico (apprendimento e condivisione di informazioni).

Un Social Network, qualunque esso sia, è principalmente definito dai contenuti. L'obbiettivo del nostro sistema è quello di invogliare gli studenti a sviluppare contenuti che approfondiscano gli argomenti trattati a lezione, incrementando la comprensione delle materie trattate e limitando lo sforzo normalmente necessario all'apprendimento. A tal fine, il docente si è premurato di utilizzare lo strumento per creare spunti di discussione reali a partire dalle definizione teoriche date a lezione, utilizzando casi di studio tratti da pubblicazioni scelte ad-hoc, per sviluppare un dibattito. In questo caso lo schema interattivo è quello tipico del blog. Altri tipi di contenuti proposti dal docente sono stati sondaggi con valutazioni numeriche relative all'interesse e alla comprensione di lezioni e seminari proposti. 
Un particolare interessante riguarda l'organizzazione dei lavori di gruppo. Uno dei due corsi interessati dal progetto CSN prevede come attività facoltativa la presentazione in classe di un approfondimento curato completamente da un gruppo di studenti. Ad ogni gruppo di volontari è stato proposto di utilizzare CSN come strumento di coordinamento e organizzazione del lavoro utilizzando la possibilità di creare sul sistema gruppi pubblicamente visibili. Questa proposta è servita a monitorare lo sviluppo del lavoro da parte degli studenti, garantendo loro un ritorno in termini di punteggio CSN accumulato a seguito della quantità di interazioni che hanno avuto tramite il sistema. Oltre a ciò, le migliori presentazioni hanno ottenuto un premio discrezionale in punti assegnato dal docente. E' stato interessante notare come, una volta che la classe ha acquisito dimestichezza col sistema, sono nati alcuni gruppi spontanei di discussione, anche su argomenti collaterali rispetto all'insegnamento. L'esempio più rilevante è costituito dalla creazione di un gruppo battezzato "keywords", creato col fine di chiarire o ampliare le definizioni tecniche date a lezione. Tale gruppo di lavoro, partito con pochi utenti e senza solleciti del docente, ha finito per comprendere la maggior parte degli iscritti al sistema. In questo si ritrovano le caratteristiche principali di un Social Network più "classico", quali l'effetto di rete che ha portato alla coalizzazione del gruppo.

\section{Definizione delle metriche proposte}

Una parte consistente del lavoro innovativo è stata la definizione di metriche per valutare l'efficacia nel processo di apprendimento del Course Social Network, sia evidenziando l'utilità percepita dagli utilizzatori, sia da parte del docente. Lo strumento, così concepito, è stato recepito bene dagli studenti? Ha portato a risultati tangibili dal punto di vista di risultati accademici? Per rispondere a queste domande abbiamo sfruttato ancora una volta la potenzialità della gamification, confrontando la classifica finale ottenuta sul sistema con l'effettiva valutazione accademica (il voto ottenuto nell'esame finale da ogni singolo partecipante al pilota). Perciò abbiamo congelato i punti CSN ottenuti dagli studenti al termine delle lezioni e su quella base abbiamo stabilito tre fasce (con soglie di definizione differenti da quelle che definiscono i livelli del gioco). Agli studenti di ciascun gruppo sarebbero stati assegnati due, uno o nessun punto bonus, in aggiunta alla valutazione (in trentunesimi, ove una valutazione di 31 significa 30 e lode) ottenuta nel test. La scelta del bonus è tale da non essere né irrilevante né così pesante da alterare la valutazione dell'apprendimento complessivo.

Le fasce di premiazione sono state identificate in base a una categorizzazione del grado di coinvolgimento degli studenti:

- $\quad$ Gli utenti che hanno ottenuto il maggior numero di punti CSN sono stati definiti "utilizzatori ad alto coinvolgimento" (UAC) ed hanno ricevuto il premio maggiore in sede di valutazione finale.

- Gli utenti definiti a "medio coinvolgimento" (UMC) sono coloro che hanno ottenuto la ricompensa intermedia, a fronte di una partecipazione attiva ma non costante alle discussione e alle attività proposte su CSN.

- L'ultima fascia include gli utilizzatori a "basso coinvolgimento", cioè coloro che non hanno ottenuto alcun bonus aggiunto al risultato del test finale (UBC).

In aggiunta a questa metrica, abbiamo raccolto le statistiche classiche che misurano l'utilizzo di un sito web, anche se non particolarmente significative per i nostri scopi. I 
risultati descritti nel prossimo paragrafo derivano principalmente dalla ricerca di correlazione tra la valutazione ottenuta in sede d'esame e i punti CSN accumulati.

\section{Risultati sperimentali}

I risultati sperimentali si riferiscono alla sperimentazione sulla classe di 80 studenti, in quanto quella relativa ai 25 studenti si è rivelata una classe troppo piccola per poter trarre risultati statisticamente significativi. Nella Figura 1 è mostrata la correlazione tra il voto ottenuto nel test finale e la classificazione della metrica proposta. Come si vede, esiste una notevole correlazione tra il voto accademico conseguito all'esame (da 18 a 31) e la partecipazione, misurata dalla nostra metrica, al progetto Course Social Network. Ovviamente, ben si sa che una correlazione non implica necessariamente una relazione di causa-effetto, ma è sicuramente un buon punto di partenza ed è la dimostrazione che l'utilizzo di CSN ha soddisfatto i principi educativi alla base del progetto. Una ulteriore prova di questa correlazione è data dalla tabella 1. Tale tabella mostra i risultati medi dei test degli studenti divisi per fascia di punteggio CSN.

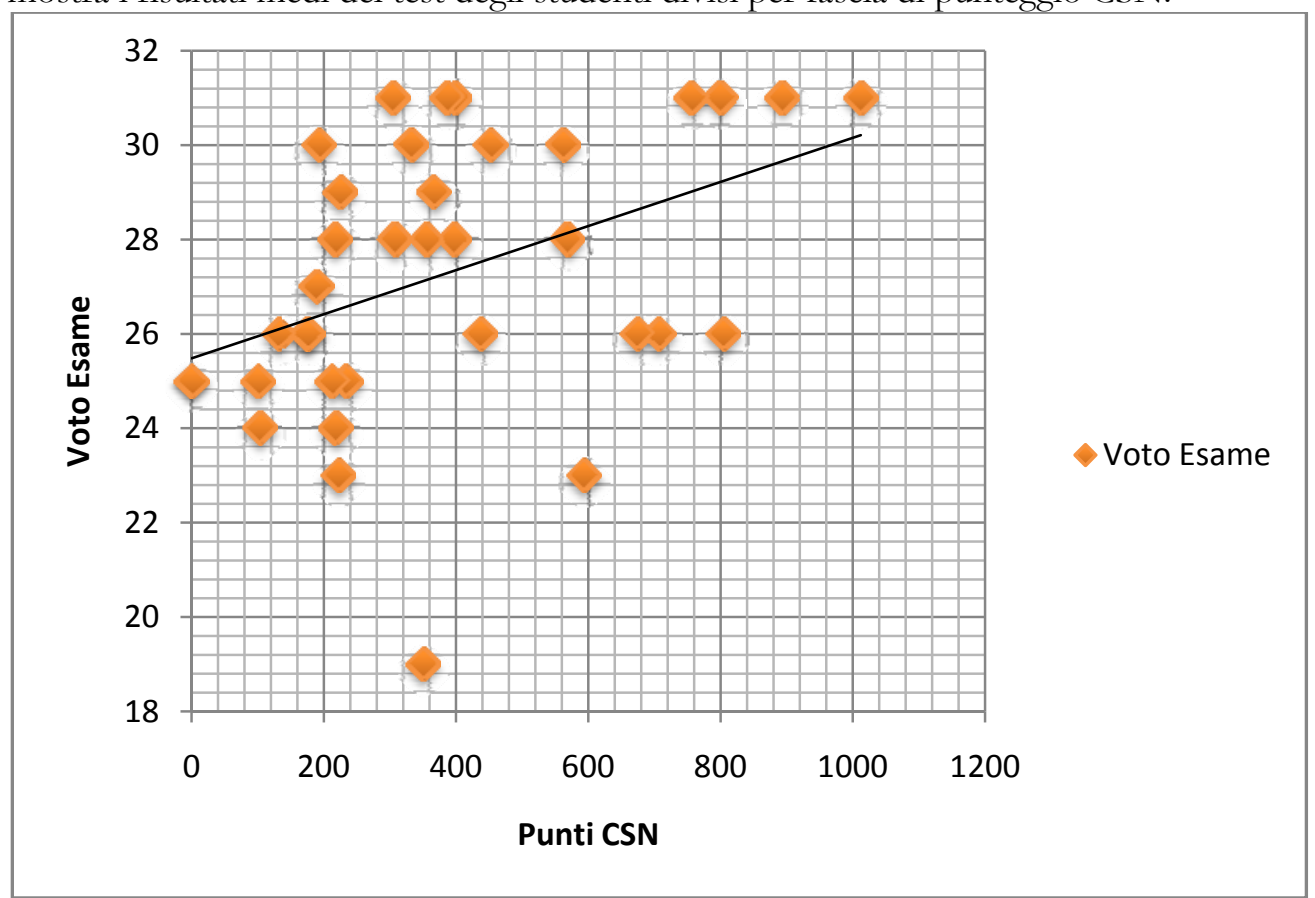

Figura 1. La correlazione tra la metrica proposta di partecipazione al Course Social Network e il voto accademico conseguito alla prova finale del corso.

Tabella 1. Le fasce di utilizzo di CSN e i relativi voti d'esame. Il campione comprende solo studenti che hanno sostenuto il test finale. Le sigle UAC, UMC e UBC indicano le tre categorizzazione del grado di coinvolgimento degli studenti.

\begin{tabular}{|l|l|l|l|l|}
\hline & $\begin{array}{l}\text { Punteggio medio } \\
\text { CSN }\end{array}$ & $\begin{array}{l}\text { Deviazione } \\
\text { Standard CSN }\end{array}$ & $\begin{array}{l}\text { Voto esame } \\
\text { medio }\end{array}$ & $\begin{array}{l}\text { Deviazione } \\
\text { Standard voto }\end{array}$ \\
\hline UAC & 853.00 & 101.86 & 30.00 & 2.23 \\
\hline UMC & 436.56 & 114.16 & 27.75 & 3.23 \\
\hline UBC & 179.40 & 57.69 & 25.87 & 1.92 \\
\hline
\end{tabular}




\section{Conclusioni}

I risultati dell'esperimento condotto, seppur preliminari visto lo svolgimento ancora in corso dell'anno accademico, mostrano, sulla popolazione studentesca che ha superato l'esame, una notevole correlazione sui risultati della metrica proposta, basata su gamification, e il voto accademico conseguito al superamento dell'esame. L'efficacia del metodo dovrà poi essere ulteriormente confermata raffrontando classi di anni accademici diversi, che sarà fatto nei prossimi anni, non solo per ampliare la statistica dei casi considerati, ma anche per escludere una correlazione casuale che potrebbe non ripetersi nel tempo.

\section{Bibliografia}

Barata, G., Gama, S., Jorge, J., \& Goncalves, D. (2013). Engaging Engineering Students with Gamification An empirical study. Games and Virtual Worlds for Serious Applications (VS-GAMES), 2013 th International Conference on (p. 1 - 8). Poole, UK: IEEE.

Berkling, K., \& Thomas, C. (2013). Gamification of a Software Engineering course and a detailed analysis of the factors that lead to it's failure. International Conference on Interactive Collaborative Learning (ICL) ( $\mathrm{p}$. 525-530). Kazan, Russia: IEEE.

Boyd, D. M., \& Ellison, N. B. (2007). Social Network Sites: Definition, History, and Scholarship. Journal of Computer-Mediated Communication, 13(1), 210 - 230.

Debatin, B., Lovejoy, J., Horn, A.-K., \& Hughes, B. (2009). Facebook and Online Privacy: Attitudes, Behaviors, and Unintended Consequences. Journal of Computer-Mediated communication, 15(1), 83 - 108.

Deterding, S., Dixon, D., Khaled, R., \& Nacke, L. (2011). From Game Design Elements to Gamefulness: Defining "Gamification". Proceedings of the 15th International Academic MindTrek Conference: Envisioning Future Media Environments (p. 9 - 15). Tampere, Finland: ACM New York, NY, USA C2011.

DiMicco, J., Millen, D. R., Geyer, W., Dungan, C., Brownholtz, B., \& Michael, M. (2008). Motivations for Social Networking at Work. Proceedings of the 2008 ACM conference on Computer supported cooperative work (p. 711 - 720). San Diego, CA: ACM.

Erenli, K. (2012). The impact of gamification: A recommendation of scenarios for education. Interactive Collaborative Learning (ICL), 2012 15th International Conference on (p. 1 - 8). Villach, Austria: IEEE.

Ford, M. (1992). Motivating Humans: Goals, Emotions, and Personal Agency Beliefs. Newbury Park, CA: SAGE Publications, Inc.

Hamari, J. (2013). Transforming homo economicus into homo ludens: A field experiment on gamification in a utilitarian peer-to-peer trading service. Electronic Commerce Research and Applications, 12(4), 236 - 245.

Hamari, J., Koivisto, J., \& Harri, S. (2014). Does Gamification Work? —A Literature Review of Empirical Studies on Gamification. 47th Hawaii International Conference on System Science (p. 3025 - 3034). Hilton Waikoloa, Big Island, Hawaii, USA: IEEE.

Huotari, K., \& Hamari, J. (2012). Defining gamification: a service marketing perspective. Proceeding of the 16th International Academic MindTrek Conference (p. 17 - 22). Tampere, Finland: ACM New York, NY, USA (C2012.

Kaplan, A. M., \& Haenlein, M. (2010). Users of the world, unite! The challenges and opportunities of Social Media. Business Horizons, 53(1), 59 - 68.

Leung, L. (2009). User-generated content on the internet: an examination of gratifications, civic engagement and psychological empowerment. New Media \& Society, 11(8), 1327 - 1347.

Lin, J., Li, Z., Wang, D., Salamantian, K., \& Xie, G. (2012). Analysis and Comparison of Interaction Patterns in Online Social Network and Social Media. Computer Communications and Networks (ICCCN), 2012 21st International Conference on (p. 1 - 7). Munich, Germany: IEEE.

Lin, K.-Y., \& Lu, H.-P. (2011). Why people use social networking sites: An empirical study integrating network externalities and motivation theory. Computers in Human Behavior, 27(3), 1152 - 1162.

Mcgonigal, J. (2011). Reality is Broken: Why Games Make Us Better and How They Can Change the World.Penguin ed.

Tsui, H.-D., Lee, C.-Y., \& Yao, C.-B. (2010). E-government, politics and Web 2.0. New Trends in Information Science and Service Science (NISS), 4th International Conference on (p. 687 - 691). Gyeongju, South Korea: IEEE.

Tuunanen, J., \& Hamari, J. (2014). Player Types: A Meta-synthesis. Transactions of the Digital Games Research Association, 1(2), 29 - 53. 